\title{
Evaluation of mouse embryos produced in vitro after electromagnetic waves exposure; Morphometric study
}

\author{
Ayoob Rostamzadeh ${ }^{1}$, Mohsen Mohammadi ${ }^{2}$, Reza Ahmadi ${ }^{3}$, Afshin Nazari ${ }^{4}$, Omar Ghaderi ${ }^{5}$, Maryam \\ Anjomshoa $^{6}$
}

${ }^{1}$ M.Sc. of Anatomy, Faculty Member, Department of Anatomical Sciences, Faculty of Medicine, Shahrekord University of Medical Sciences, Shahrekord, Iran

${ }^{2}$ Ph.D. of Pharmaceutical Biotechnology, Assistant Professor, Department of Pharmaceutical Biotechnology,

Faculty of Pharmacy, Lorestan University of Medical Sciences, Khorramabad, Iran

${ }^{3}$ Ph.D. Candidate of Clinical Biochemistry, Department of Clinical Biochemistry, Faculty of Medicine, Iran University of Medical Sciences, Tehran, Iran

${ }^{4}$ Ph.D. of Physiology, Assistant Professor, Department of Physiology, Faculty of Medicine, Lorestan University of Medical Sciences, Khorramabad, Iran

${ }^{5}$ Ph.D. Candidate of Pharmaceutical Biotechnology, Department of Pharmaceutical Biotechnology, Tehran University of Medical Sciences, Tehran, Iran

${ }^{6}$ Ph.D. of Anatomy, Assistant Professor, Department of Anatomical Sciences, Faculty of Medicine, Shahrekord University of Medical Sciences, Shahrekord, Iran

\section{Type of article: Original}

\begin{abstract}
Introduction: Today, the use of electromagnetic waves in medical diagnostic devices, such as magnetic resonance imaging (MRI), has increased, and many of its biological effects have been reported. The aim of the present study was to assess the biological effects of 1.5 Tesla (T) magnetic resonance imaging (MRI) on fertility and reproductive parameters.

Methods: Eighty adult male and female NMRI mice (NMRI: Naval Medical Research Institute) of age 6-8 weeks were studied and randomly divided into two study and control groups. After confirmation of pregnancy, the mice in the study group were exposed to the MRI (1.5 T) machine's waves over the next three weeks, once a week for 36 minutes. One day and thirty-five days after the last radiation, the mice were killed in order to do the in vitro fertilization (IVF) by neck beads' displacement and the impact on the evolution of embryos, and its quality was studied. Data were analyzed using SPSS version 20 and the significance level of less than 0.05 was considered.

Results: Embryo morphometry showed that the total diameter and the cytoplasm diameter of the study group embryos suffered significant reduction compared to the control group, 1 day after the last irradiation $(\mathrm{p}<0.05)$, but the diameter of the perivitelline space of this group's embryos had a significant increase $(p<0.05)$. The qualitative results during 35 days after irradiation showed that morphologically parameters of the embryos in the study group had no significant differences from the control group.

Conclusion: Exposure to MRI irradiation can transiently disturb the development of mouse embryos and fertility, but these effects are reversible 35 days after the last irradiation.

Keywords: MRI (Magnetic resonance imaging), fertility, embryo morphometry
\end{abstract}

\section{Introduction}

The human population is exposed significantly to natural and man-made sources of ionizing and non-ionizing radiation (1). The most important manmade equipment, magnetic resonance imaging (MRI), has resulted in rapid development of technologies in medicine (2). This method has been used to evaluate functional or physiological

\section{Corresponding author:}

Assistant Professor Dr. Maryam Anjomshoa, Department of Anatomical Sciences, Faculty of Medicine, Shahrekord University of Medical Sciences, Shahrekord, Iran. Fax: +983813334911, E-mail; anjomshoa.m@gmail.com Received: September 27, 2015, Accepted: December 04, 2015, Published: January 2016 iThenticate screening: November 16, 2015, English editing: January 02, 2016, Quality control: January 05, 2016 (C) 2015 The Authors. This is an open access article under the terms of the Creative Commons Attribution-NonCommercialNoDerivs License, which permits use and distribution in any medium, provided the original work is properly cited, the use is non-commercial and no modifications or adaptations are made. 
status of tissue (3). MRI seems to be the most suitable examination technique to assess a wide range of human abnormalities, such as central nervous system (CNS), cardiovascular system, musculoskeletal system, and reproductive system (4). Furthermore, MRI can be used in cases in which ultrasound is not conclusive for pregnant women (5). Technological advances in MRI (higher static fields, faster gradients, stronger RF transmitters) have occurred rapidly, and many questions regarding the safety of these developments remain unanswered (5-7). Compared to the X-ray based medical diagnostic techniques (e.g., general radiography, positron emission tomography (PET), and computed tomography (CT)), MRI does not involve exposure to ionizing radiation. MRI uses three main components to produce images from inside the body, i.e., static (main) magnetic field (B0), $0 \mathrm{~Hz}$; time-varying gradient electromagnetic (TVM) fields, 3-3000 Hz; and radiofrequency (RF) electromagnetic fields, $10-400 \mathrm{MHz}$ (5-8). The static or main magnetic field is used to align the nuclei in the patient's body. This powerful static magnetic field is always present even when the MR scanner is not imaging. The biological effects of static magnetic fields are one of the most controversial topics in the field of MR safety $(7,8)$. It has been reported that several structures are influenced by the static magnetic fields, such as the retina, pineal gland, and some cells in the Para nasal sinuses. Moreover, other biological disorders, including vertigo, difficulty with balance, nausea, headaches, numbness and tingling, phosphines, and unusual taste sensations, were noted at $1.5 \mathrm{~T}$ exposures. Moreover, the static field extends beyond the confines of the scanner bore, and this is called the fringe field. This field is important with regard to interference with vital medical devices, such as cochlear and cardiac implants (2, 4, 7-9). TVM fields induce an electric field, which used to alter the main magnetic field and allow the signal from the patient to be spatially encoded into a picture. The magnitude of the switched MF gradient and its time derivative is dependent on the applied pulse sequence parameters. The gradients are pulsed within and between RF excitation pulses and turn on/off very quickly during scanning, causing the knocking noise associated with MRI.

The main safety concerns with the TVM fields are acoustic noise, induced electric fields, and circulating currents in conductive tissues (4-6). A radio frequency pulse (a short burst of an electromagnetic radiation) is used in MRI to "excite" tissue protons by an exchange of energy. These protons give a signal in return. The RF spectrum typically used in MRI covers the same frequencies that are used by radio stations (around $100 \mathrm{MHz}$ ). The RF field is usually created by a body coil integrated into the scanner that produces a circularly-polarized MF with a frequency of approximately $42 \mathrm{MHz} / \mathrm{T}$, known as B1 (2, 4-7). The thermal characteristics of different organs and organ parts are different. Limbs dissipate thermal energy more rapidly than the internal parts of the abdomen. The testes, which are separated from the main volume of the body, are regarded as heat sensitive (10). Generally, an increase of $1{ }^{\circ} \mathrm{C}$ is acceptable to a normal healthy body $(2,10)$. For healthcare staff (nurses, technicians and radiologists), the static magnetic field from MRI scanners is of special concern because the field is always turned on during their shifts. Highest exposure occurs in the direct proximity to the magnet's housing $(7,8)$. Healthcare workers are exposed to static magnetic fields while attending patients before and after examination and also while operating the scanner's console situated on the housing of the magnet (4-6, 8). Exposure to gradient and RF pulses is possible only during an examination; it affects workers in special cases only, e.g., during the so-called dynamic examinations or in emergencies $(5,7,8)$. Potential health risks can be attributed to exposure with each type or with the combination of the three different magnetic field components used in MRI. In some cases, the potential exists for medical staff to actually be inside the magnetic field for hours for each procedure and thus potentially for a significant period of time per year $(2,6,7)$. Wile'n and de Vocht estimated that each scanner will involve about five workers from different disciplines, such as radiographers, nurses, anesthetists, technicians, engineers, and cleaners and that these people may be exposed to various electromagnetic fields (EMF) during procedures (11). In addition, Kunt et al. reported in 2011 that the staff working at the MRI department have significantly lower bone mineral density than the average value in society, and this could be due to reduced synthesis of osteoblasts, resulting in the induction of apoptosis and death (12). Other studies have shown that people who are under the MRI routine experience a significant reduction in permeability and elasticity of the membranes of their red blood cells (13). In some centers, procedures, such as interventional MRI, also involve other staff groups, such as surgeons and cardiologists. Also, field surveys among nurses, engineers, and radiographers who routinely work adjacent to MRI scanners describe various symptoms, such as vertigo, nausea, and illusion of movement.

To date, there have been reports from in vivo and in vitro experiments where a single MRI scan may have genotoxic effects. However, this must be investigated further. Due to the active shielding, the field decreases quickly with increasing distance from the scanner, producing a large gradient of the static field so that the field of 1.5-3 $\mathrm{T}$ scanners may only become significant within about $0.5-1.0 \mathrm{~m}$ from the bore opening. 


\section{Material and Methods}

\subsection{Animals and wave exposure}

In this study, 40 adult male and 40 adult female NMRI mice of age 6-8 weeks and approximate weight of $30 \pm 5$ grams (from Pasteur Institute, Tehran, Iran) were studied. The mice were kept for two weeks in the animals' lab at standard conditions, i.e., temperature of 23 to $25{ }^{\circ} \mathrm{C}$, humidity of 50 to $55 \%$, a 12 -hour light cycle, and easy access to food and water for compatibility with the environment. The mice were divided randomly into two groups of equal numbers, i.e., a study group and a control group. To confirm the fertility of both groups, male mice were placed near the female mice overnight. The next morning at about 9:00 A.M., the female mice were studied for having vaginal plate and, if the results were positive, they were kept in a separate cage until the time of delivery. After confirmation of pregnancy, the mice in the study group were exposed to the MRI scanner's waves over the next three weeks, once a week for 36 minutes. For the purpose of irradiation, we used a superconductor Siemens device (Magnetom ESSENZA, Germany) with 1.5 Tesla intensity and the frequency of $64 \mathrm{MHz}$. For the MRI irradiation, first, the mice were placed in a plastic container with a cardboard lid (to avoid any disrupting electromagnetic waves), and, then, they were placed in the center of the machine. The mice in the control group also were transferred to the MRI's center in a plastic container, but they were kept away from the magnet room. One day after the last radiation, 20 mice were killed in order to do the IVF by neck beads' displacement, and the impact on the evolution of embryos and their quality were studied (Figure 1).

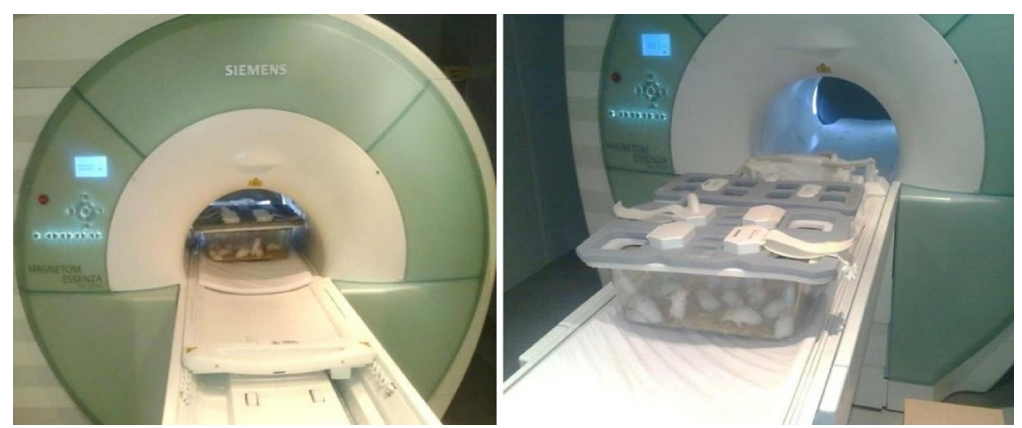

Figure 1. Transfer of mice to the MRI scanner in a plastic container

\subsection{Sperm collection}

In order to collect sperm, the epididymis tail and vas deferens of the mice were dismembered and put into a Petri dish containing HTF in vitro that had already been balanced in an incubator. Then, the tail and the epididymis and the vas deferens were split into smaller pieces and were placed for 1 hour inside the incubator (Memmert Co., Germany) at $37{ }^{\circ} \mathrm{C}$ and $5 \% \mathrm{CO}_{2}$ for capacitation.

\subsection{Oocyte collection}

For the induction of oocyte development and reproduction in each mouse, five international units (IU) of PMSG hormone (Folligon, Intervet, Netherland) was injected into the peritoneum. For the purpose of inducing the ovulation process, 48 to 50 hours after the injection of the hormone PMSG, 5 units of HCG (Chorulon, Intervet, Netherland) was injected in intraperitoneal form to each mouse. Twelve hours prior to the isolation of oocytes, the HTF and KSOM in vitro were dropped and covered with mineral oil and maintained in incubators. On the isolation day and 12 hours after hCG hormone injections, the female mice were killed by neck beads' displacement, and the set of eggs and cumulus cells were collected from the ampulla of the uterine tubes of both sides and placed into the Petri dish containing HTF in vitro that had earlier been balanced in an incubator.

\subsection{In Vitro Fertilization}

At this point, the sperm of the experimental group (radiation exposed) and the control group underwent in-vitro fertilization, such that an equal number of sperm from both groups, -which had been gathered at sides of the drops in swim up way, were removed by sampler and added to drops containing oocytes. Then the oocyte and spermcontaining environment was put in the incubator for 4 to $5 \mathrm{hr}$. Then, the oocytes likely of insemination were transferred to a Petri dish containing 5 drops of KSOM in vitro. Oocytes were finally transferred to the fifth in the middle of the Petri dish after a wash in four side drops. Twenty-four hours after insemination, the number of twocell (and probably four-cell) embryos were counted and recorded by a stereomicroscope (Zeiss Co., Germany). Monitoring the evolution of the embryos continued until the blastocyst stage was reached in the following days. To 
check the reversibility status of the possible effects of MRI waves on the fertility of the mice, all the steps listed above were repeated for 20 mice, 35 days after irradiation.

\subsection{Grading of embryo}

The morphology of two blastomer embryos were divided into four grades of A, B, C, and D (14). Grade A with equal blastomers, round, with no fragmentation, smooth cytoplasm, and bright yellow zona; grade B with slightly different blastomers in size, up to $10 \%$ fragmentation with granules in cytoplasm; grade $\mathrm{C}$ with unequal blastomers, up to $50 \%$ fragmentations and large granules and vacuoles in cytoplasm; and grade $\mathrm{D}$ with blastomers of unequal size, extreme fragmentation, with dark and large granules and presence of vacuoles in cytoplasm (Figure 2).

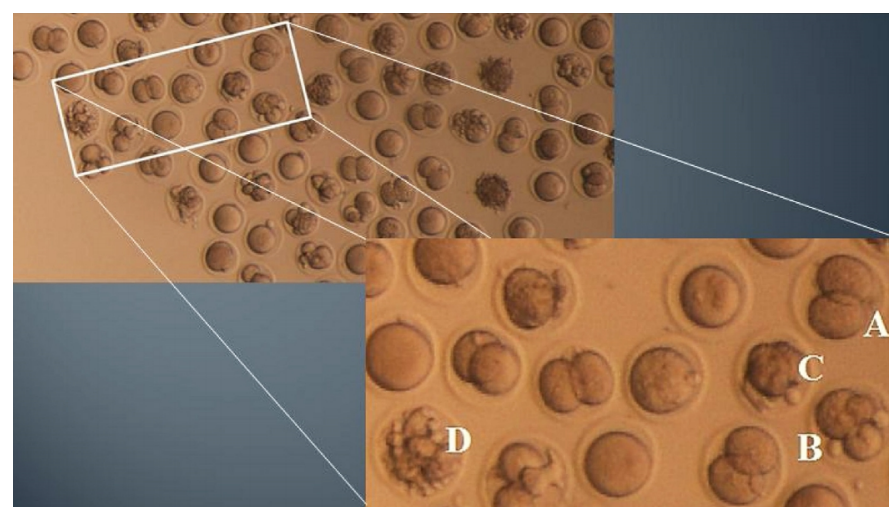

Figure 2. Bolto scale for quantitative assessment of 2-cell embryos on culture medium

\subsection{Embryo Morphometry}

After taking images by the inverse microscopes and analyzing them with Nikon software, the images were entered into the Canon software Image and necessary measures were taken. The resulting embryos were evaluated in terms of overall diameter, the diameter of the cytoplasm and the thickness of the zona pellucida. The longest diameter of embryo was considered the basic diameter for embryo morphometry, and the cytoplasm diameter and zona pellucida thickness also were measured on the basis of the same axis. The thickness of the zona pellucida was measured such that the thicknesses of both sides were added together, and the total was considered to be the true thickness. Also, the diameter of the perivitelline space was measured approximately by the overall diameter minus the thickness of the cytoplasm diameter minus the total thickness of zona pellucida (Figure 3).

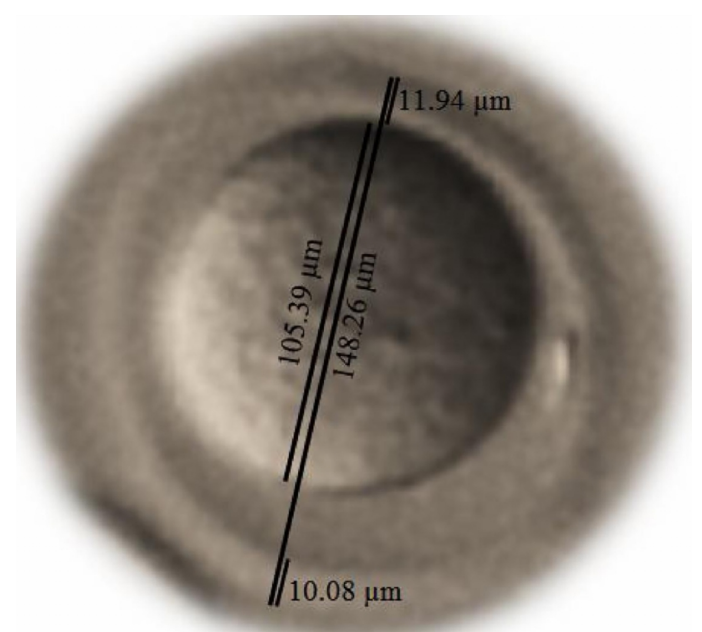

Figure 3. Use of the public image analysis software program Image $\mathbf{J}$ for morphometric analysis of embryos: The largest diameter was selected as a base for all quantitative embryo assessment in all groups (10 micrometer-scale). The diameter of the perivitelline space was approximately measured by the overall diameter minus the thickness of the cytoplasm diameter minus the total thickness of zona pellucid. 


\subsection{Statistical analysis}

To compare the speed of cleavage divisions and the number of embryos, one-way ANOVA and variance analysis were used, and the results were expressed as numbers and percentages. To check the quality of the embryos and their morphometry, the chi-squares test was used. All statistical tests were carried out using SPSS software, version 20 (IBM SPSS), and the significance level of less than 0.05 was considered. The charts were drawn using Excel software.

\subsection{Ethics Approval}

All experimental procedures involving animals were approved by the Ethics Committee of Kurdistan University of Medical Sciences.

\section{Results}

\subsection{One day after irradiation}

The quantitative results obtained by the Tukey test demonstrated that the rate of evolutionary progress of two-cell embryos in the radiation-exposed group had a significant reduction in comparison with the control group $(\mathrm{p}<0.05)$. The results also showed that the number of embryos that reached the blastocyst stage in the experimental group had a significant reduction in comparison with the control group $(\mathrm{p}<0.05)$, and the number of the dead embryos also had a statistically significant increase compared to the control group $(\mathrm{p}<0.05)$ (Table 1) (Figures 4 and 5). The results of the quality control of the two-cell embryos, based on the bolto scale 1 day after the last radiation, showed that the number of embryos with grade A in the study group was reduced significantly compared to the control group $(32.5 \%)(\mathrm{p}<0.05)$. In addition, the embryos with grade $\mathrm{B}$ and $\mathrm{D}$ in the study group had a significant increase in comparison with the control group $(12.50$ to $13.33 \%$, respectively) $(p<0.05)$ (Table 2$)$. However, the results of embryo morphometry by the Image $\mathbf{J}$ software showed that the total diameter and the cytoplasm diameter of the study group embryos suffered significant reduction compared to the control group 1 day after the last irradiation $(\mathrm{p}<$ $0.05)$, but the diameter of the perivitelline space of this group's embryos had a significant increase $(\mathrm{p}<0.05)$ (Table $3)$.

Table 1. Quantitative data of embryo cleavage in control and study groups several days after MRI irradiation

\begin{tabular}{|c|c|c|c|c|c|c|c|}
\hline \multicolumn{2}{|l|}{ Groups/Days } & \multicolumn{2}{|c|}{ Two-cell } & \multicolumn{2}{|c|}{ Blastocyst } & \multicolumn{2}{|c|}{ Dead cell } \\
\hline & & $\mathrm{n}$ & $\%$ & $\mathrm{n}$ & $\%$ & $\mathrm{n}$ & $\%$ \\
\hline \multirow[t]{4}{*}{ Control $(\mathrm{n}=118)$} & Day 1 & 80 & 67.79 & 0 & 0 & 10 & 8.47 \\
\hline & Day 2 & 8 & 6.77 & 0 & 0 & 14 & 11.86 \\
\hline & Day 3 & 6 & 5.08 & 19 & 16.1 & 18 & 15.25 \\
\hline & Day 4 & 2 & 1.69 & 60 & 50.84 & 30 & 25.42 \\
\hline \multirow[t]{4}{*}{ Study $(\mathrm{n}=92)$} & Day 1 & $47^{\mathrm{a}}$ & $51.08^{\mathrm{a}}$ & 0 & 0 & 16 & 17.39 \\
\hline & Day 2 & 7 & 7.6 & 0 & 0 & $23^{c}$ & $25^{\mathrm{c}}$ \\
\hline & Day 3 & 3 & 3.26 & $4^{b}$ & $4.34^{\mathrm{b}}$ & $30^{c}$ & $32.6^{\mathrm{c}}$ \\
\hline & Day 4 & 3 & 3.26 & $23^{\mathrm{b}}$ & $25^{\mathrm{b}}$ & $38^{\mathrm{c}}$ & $41.3^{\mathrm{c}}$ \\
\hline
\end{tabular}

${ }^{\mathrm{c}} \mathrm{p}<0.05$ vs. Control in Dead cell groups, ${ }^{\mathrm{a}} \mathrm{p}<0.05$ vs. Control in Two-cell groups, ${ }^{\mathrm{b}} \mathrm{p}<0.05$ vs. Control in Blastocyst groups.
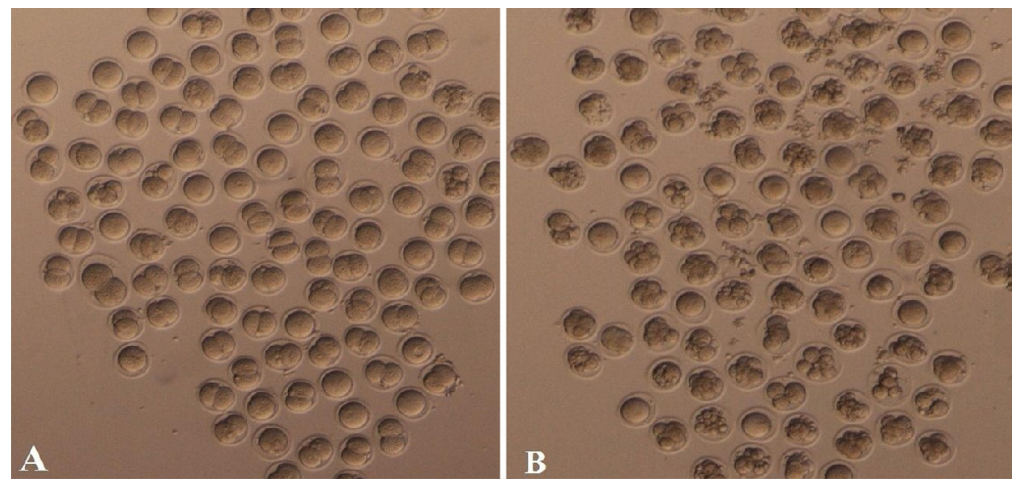

Figure 4. Stereomicroscope imaging of the fetus in KSOM medium the second day after IVF: A) Evolution of embryos is favorable in the control group; B) mbryos in the study groups represent two-cell arrest; after arrest, the two-cell embryo will degenerate and experience fragmentation. 

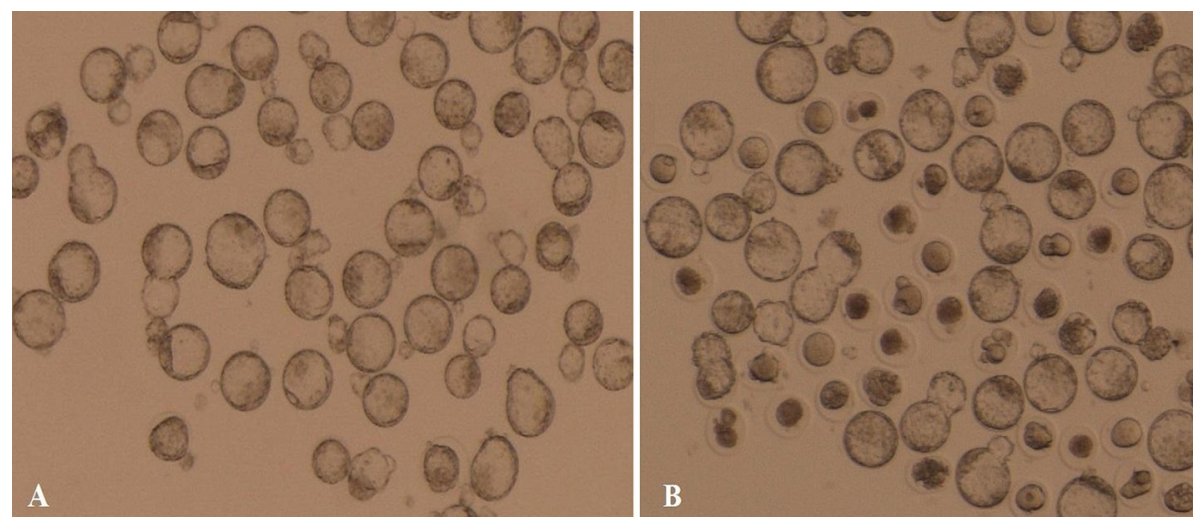

Figure 5. Stereomicroscope imaging of the blastocysts (one day after irradiation) in KSOM medium 4th day after IVF: A) A large number of embryos progress to the blastocyst stage and had no morphological abnormalities; B) A large number of embryos is arrested at the one- to two-cell stage and some of them became degenerated and experienced cytoplasmic vacuolization, fragmentation, and cell death with abnormal and pyknotic appearance.

Table 2. Effects of one day and thirty-five days after the last MRI irradiation on the quality of the two-cell embryos in the control and study groups

\begin{tabular}{|l|l|l|l|l|l|}
\hline \multicolumn{2}{|l|}{ Groups } & \multicolumn{2}{l|}{1 day after irradiation } & \multicolumn{2}{l|}{35 days after irradiation } \\
\cline { 3 - 6 } & & Control & Study & Control & Study \\
\hline \multirow{2}{*}{ Grade A } & $\mathrm{n}$ & 87 & ${ }^{a} 48$ & 86 & 71 \\
\cline { 2 - 6 } & $\%$ & 72.50 & ${ }^{a} 40.00$ & 71.66 & 59.16 \\
\hline \multirow{2}{*}{ Grade B } & $\mathrm{n}$ & 15 & ${ }^{\mathrm{a}} 31$ & 17 & 21 \\
\cline { 2 - 6 } & $\%$ & 12.50 & ${ }^{\mathrm{a}} 25.83$ & 14.17 & 17.50 \\
\hline Grade C & $\mathrm{n}$ & 10 & 18 & 8 & 13 \\
\cline { 2 - 6 } & $\%$ & 8.33 & 15.00 & 6.67 & 10.84 \\
\hline \multirow{2}{*}{ Grade D } & $\mathrm{n}$ & 8 & ${ }^{\mathrm{a}} 23$ & 9 & 15 \\
\cline { 2 - 6 } & $\%$ & 6.67 & ${ }^{\mathrm{a}} 19.17$ & 7.50 & 12.50 \\
\hline
\end{tabular}

${ }^{\mathrm{a}} \mathrm{p}<0.05$ vs. Control, 1 day after irradiation

Table 3. Morphological data of embryo in the control and study groups one day and thirty-five days after the last MRI irradiation

\begin{tabular}{|l|l|l|l|l|}
\hline \multirow{2}{*}{ Measurements $(\mu \mathrm{m})$} & \multicolumn{2}{|l|}{ 1 day after irradiation } & \multicolumn{2}{l|}{ 35 days after irradiation } \\
\cline { 2 - 5 } & Control & Study & Control & Study \\
\hline Total fetal diameter & $147.82 \pm 0.12$ & ${ }^{\mathrm{a}} 112.22 \pm 1.66$ & $145.36 \pm 1.45$ & $140.70 \pm 0.78$ \\
\hline Cytoplasm diameter & $120.45 \pm 0.54$ & ${ }^{\mathrm{a}} 80.36 \pm 1.32$ & $117.34 \pm 0.62$ & $114.04 \pm 1.16$ \\
\hline Zona pellucida thickness & $19.14 \pm 0.85$ & $16.79 \pm 1.06$ & $19.03 \pm 1.36$ & $16.74 \pm 0.44$ \\
\hline Perivitelline space diameter & $8.64 \pm 0.06$ & ${ }^{\mathrm{a}} 15.89 \pm 1.04$ & $8.58 \pm 0.42$ & $8.76 \pm 0.35$ \\
\hline
\end{tabular}

${ }^{\mathrm{a}} \mathrm{p}<0.05$ vs. Control, 1 day after irradiation

\subsection{Thirty-five days after irradiation}

The qualitative results during this period showed that there was not a statistically significant difference in the level of evolutionary progress of two-cell embryos as well as the number of embryos that have reached the blastocyst stage between the study group and the control group $(\mathrm{p}=0.68$ and $\mathrm{p}=0.57$, respectively). The number of dead in the study group was not significantly different from the number in the control group $(p=0.93)$ (Table 4). Also, the quality control of the two-cell embryos based on the bolto scale, 35 days after the last irradiation, represented that there was not a significant difference between the number of grades in the study group and the control group $(\mathrm{p}=$ 0.74). However, the results of the embryo morphometry showed that the morphological parameters of the embryos, i.e., the overall diameter, the cytoplasm diameter, the thickness of the zona pellucida, and the diameter of the 
perivitelline space in the study group after 35 days from the last irradiation, had no significant difference compared to the control group $(\mathrm{p}=0.46)$ (Tables 2 and 3$)$.

Table 4. Quantitative data of embryo cleavage in control and study groups thirty-five days after MRI irradiation

\begin{tabular}{|l|l|l|l|l|l|l|l|}
\hline Groups/Days & \multicolumn{2}{l|}{ Two-cell } & \multicolumn{2}{l|}{ Blastocyst } & \multicolumn{2}{l|}{ Dead cell } \\
\cline { 3 - 9 } & & $\mathrm{n}$ & $\%$ & $\mathrm{n}$ & $\%$ & $\mathrm{n}$ & $\%$ \\
\hline Control (n=67) & Day 1 & 48 & 71.64 & 0 & 0 & 4 & 5.97 \\
\cline { 2 - 9 } & Day 2 & 3 & 4.47 & 0 & 0 & 6 & 8.95 \\
\cline { 2 - 9 } & Day 3 & 2 & 2.98 & 8 & 11.94 & 12 & 17.91 \\
\cline { 2 - 8 } & Day 4 & 0 & 0 & 38 & 56.71 & 27 & 40.29 \\
\hline Study (n=71) & Day 1 & 46 & 64.78 & 0 & 0 & 6 & 8.45 \\
\cline { 2 - 9 } & Day 2 & 6 & 8.45 & 0 & 0 & 10 & 14.08 \\
\cline { 2 - 8 } & Day 3 & 3 & 4.22 & 6 & 8.45 & 18 & 25.35 \\
\cline { 2 - 8 } & Day 4 & 1 & 1.4 & 37 & 52.11 & 31 & 43.66 \\
\hline
\end{tabular}

\section{Discussion}

The findings of this study demonstrated that the MRI irradiation with 1.5 Tesla intensity and $64 \mathrm{MHz}$ frequency for 36 minutes (once a week for three consecutive weeks) 1 day after the last irradiation resulted in the reduction of the number of two-cell embryos. Our findings also showed that after MRI irradiation the number of embryo that have reached blastocyst stage, the number of embryos with grade $\mathrm{A}$, the total diameter and the cytoplasm diameter were increased. Also the number of dead embryos in vitro, the number of embryos with grade B and D and the diameter of the perivitelline space were increased. But after 35 days from the last irradiation, these parameters were significantly reversible. There are many other studies on the electromagnetic effects resulting from other sources that are conducted separately on fertility that include a constant weak magnetic field, magnetic field variable with time and RF field. These electromagnetic fields are respectively preset in equipment, such as high-pressure cables, home computers, and mobile phones. Wdowiak et al.'s study in 2007 showed that the mobile phone radiation in males led to lower sperm counts, lower sperm motility, more abnormal sperm, and lower fertility (15), which is in line with the results of the present study. In 2011, Saadeldin et al. also studied the effects of the 1-Gauss magnetic field radiation with a frequency of $50 \mathrm{~Hz}$ for 21 days on the fertility of male rats. In this study, one of the groups was investigated immediately after irradiation, and the other group was investigated 48 days after the last irradiation (period of rat spermatogenesis), and they found that the weight of the testes and the number of sperm, 1 day after irradiation, in the irradiated group had significant reduction compared to the control group, while after 48 days these changes returned to the normal state. The researchers showed that the magnetic field of the testicular function was biologically toxic and led to the induction of oxidative stress on the process of spermatogenesis (16). The results of the study of Saadeldin et al. in terms of fertility change 1 day after this irradiation and its reversibility after a period of spermatogenesis (48 days) were consistent with the results of our study. According to Desai et al.'s report, radiofrequency waves with effects on the sperm's plasma membrane enzymes cause the production of cellular superoxidize in semen and decrease phosphokinase enzyme $\mathrm{C}$ activities (plays a role in the sperm motility) and reduce the ability to survive, the number of sperm, and their mobility (17). Therefore, this report could explain the decrease in the number of two-cell embryos, the number of embryos that have reached blastocyst stage, the number of embryos with grade A, total diameter and the cytoplasm diameter in the present study. Studies have shown that, in addition to thermal effects, RF waves have non-thermal effects, which can be caused by free radical production $(4,18,19)$. In turn, free radicals cause phosphorylation and activation of some of the messenger proteins, such as histone kinases and keratin kinases, and, due to these changes, the speed of production of ROS increases significantly and activates the path of caspase-3 in sperm cells as a result of which the death of apoptotic cells during spermatogenesis or sperm maturity period and affects fertility (20). The human body has a defense system to cope with free radicals that is known as anti-oxidants system. An imbalance between the amount of free radicals produced and anti-oxidants' capacity causes oxidative stress. The most important free radicals in human semen include anion superoxide radical, hydrogen peroxide, and hydroxyl radical. These free radicals are normally produced during oxygen metabolism (17, 19-21). Although under physiologic conditions, lower amounts of active oxygen species are necessary for natural sperm functioning, such as capacity-taking, acrosome reaction, mobility power, fertility power, and fertilization, but the existence of excessive amounts of ROS can cause serious damage in the sperms as non-thermal radiofrequency radiation effects are one of the reasons for the production of ROS (22). During the process of capacity-taking, the calcium intracellular levels, ROS, and enzyme tyrosine-kinase activity increases, leading to increased adenosine monophosphate (cAMP). Increasing cAMP results in facilitation of 
sperm's hyper-activation during which point their mobility increases. Only sperm in which the process of capacitytaking has taken place are fit for the phenomenon of activation and the promotion of the acrosome reaction that eventually leads to the acquisition of the ability to fertilize. Active oxygen species are involved in the interaction between the sperm and the egg, such that the phenomenon of lipid peroxidation caused by amounts of ROS leads to changes in the sperm's membrane and facilitation of sperm-egg binding. However, there is evidence that an excessive amount of ROS led to a sperm-egg unbinding and no or faulty fertilization that will lead to failure in reproduction. This factor can be an explanation for the reduction of the number of embryos in vitro and also changes of morphometric embryo parameters, such as the total diameter and cytoplasm diameter in the present study (19-21, $23,24)$. However, the DNA sperm molecule also may be damaged during the process of oxidative stress. Bases and phosphodiester bonds in DNA are prone to peroxidation damage caused by free radicals that may lead to disorders, such as changes of bases, production of positions with freebases, bases' removal, transverse connections, and chromosomal rearrangements. The process of apoptosis can help the removal of abnormal cells and prevention from the production of too many of them. Free radicals have the ability to stimulate the start of apoptosis reactions' process. Any damage in DNA can accelerate the process of apoptosis, which ultimately leads to reduced sperm count, increased embryonic mortality, and probably reduced cell survival to the different stages of evolution, such as blastocyst stage that could be observed in the results of the present study (22-25). Another potential stressful resource in our study was the noise caused by the MRI machine's gradients during the scan. According to some reports, producing noise and radiofrequency radiation simultaneously can be an important factor for the reduction of sperm's parameters, such as the number of them, their survival and morphology, as well as the reduction of antioxidant capacity and power to reproduce for Wistar rats (26). The value of 1.5 Tesla MRI machine noise usually varies between 101.8 to $111.7 \mathrm{~dB}$ during a typical scanning session (27). According to the results of Pramanik et al.'s study, producing $90 \mathrm{~dB}$ noise for 5 hours a day to the Albino rat results in significant reduction of sperm morphology and sperm count as well as a reduction of testosterone levels and ultimately reduced fertility (28). Similarly, in the present study, the reduced power of fertility was observed in vitro that could be caused by the negative effect of gradients' noise on the antioxidant capacity as well as disruption of the sperm-oocyte connection. Today, many scholars believe that the basis of cellular and biological injuries must be searched in membrane changes. Since alkaline phosphatase enzyme (ALP) plays an important role in many cellular processes, and its activity changes by the ratio of total cholesterol to cell membrane phospholipid, the damage of cell membrane would lead to higher release of this enzyme in inter-cell fluids and serums. Alkaline phosphatase enzyme intervenes in the local adjustment of blood flow through controlling the AMP nucleotide level and on cell through intervention in the transfer of calcium from the cell membrane and its function as a Ca-Mg-ATPase and intervention in the activity of membrane enzymes in the cell. So the energy from the waves through affecting the value of alkaline phosphatase enzyme causes the destruction of the cell membrane, the induction of apoptosis, and finally increases cell death (29). In the present study, the number of two-cell embryos significantly reduced, and there was a significant increase of dead embryos 1 day after irradiation, which, according to the results of El-Gebaly et al., can be caused by sperm cell membrane ruptures and cell apoptosis induction. Bernabò et al. reported that irradiation to wild hogs with $50 \mathrm{~Hz}$ electromagnetic fields for 12 hours for 6 days caused the destruction of acrosome, reduction of the capacity of acrosome reactions, changes in sperm functions, reduction of the occurrence of sperm fertility process, and less power of fertilization (30). In the present study, the number of two-cell embryos in the study group was reduced, which probably could be attributed to the damaging effects of the TVM fields with MRI time (the equivalent of ELF waves) that influenced the chromosome and therefore caused sperm death and reduced sperm count. It also affected sperm morphology and structure and decreased the capacity of the sperm in the fertilization process and led to a reduction of the number of oocyte fertilization and two-cell embryos. In the present study, although irradiation to male mice with 1.5 Tesla MRI machines for 36 minutes (once every week for three weeks) caused destructive effects in fertility sperm power, embryo development in vitro, and also the quality of the embryos and fetal morphometric parameters, such as total diameter, cytoplasm diameter, and the thickness of the zona pellucida one day after irradiation. All of these effects were eliminated after a period of spermatogenesis. But the important issue here is the cofactors' discussion that can have a synergy effect (the cumulative effect of the genetic damage, particularly genetic damages) on the level of damages they have caused. This issue is very important, because several groups of people also are exposed to the irradiation of the three MRI fields, including equipment personnel, medical imaging researchers, the crew of the division, the machines' technical operators (during contrast material injection in dynamic tests), and interventional surgeons $(7,8)$. In the past, the primary safety concerns have focused on patients, since technologists and physicians are only transiently near the magnet. With the advent of interventional MRI, interventional procedures are completed in or adjacent to the magnet, and the potential effects of acute and/or long-term exposure to the medical staff are much greater. 


\section{Conclusions}

The results of this study showed that the exposure to MRI irradiation of $1.5 \mathrm{~T}$ transiently can disturb the development of mouse embryos and fertility, but these effects are reversible after 35 days from the last irradiation. It is recommended that further research should be conducted on animal models as well as molecular genetic studies.

\section{Acknowledgments:}

This project was supported financially by Kurdistan University of Medical Sciences, Sanandaj, Iran, with grant number KA/93/931. The authors thank the Vice Chancellor of Research at Kurdistan University of Medical Sciences.

\section{Conflict of Interest:}

There is no conflict of interest to be declared.

\section{Authors' contributions:}

All authors contributed to this project and article equally. All authors read and approved the final manuscript.

\section{References}

1) Wakeford R. The cancer epidemiology of radiation. Oncogene. 2004;23(38):6404-28. doi: 10.1038/sj.onc.1207896. PMID: 15322514.

2) Hartwig V, Giovannetti G, Vanello N, Lombardi M, Landini L, Simi S. Biological effects and safety in magnetic resonance imaging: a review. Int J Environ Res Publ Health. 2009;6(6):1778-98. doi: 10.3390/ijerph6061778. PMID: 19578460, PMCID: PMC2705217.

3) Gawryluk JR, Mazerolle EL, D'Arcy RC. Does functional MRI detect activation in white matter? A review of emerging evidence, issues, and future directions. Front Neurosci. 2014;8. doi: 10.3389/fnins.2014.00239. PMID: 25152709, PMCID: PMC4125856.

4) Formica D, Silvestri S. Biological effects of exposure to magnetic resonance imaging: an overview. BioMedical EngineeringOnline. 2004;3(1):11. doi: 10.1186/1475-925X-3-11. PMID: 15104797, PMCID: PMC419710.

5) Kangarlu A, Robitaille P-ML. Biological effects and health implications in magnetic resonance imaging. Concepts Magn Resone. 2000;12(5):321-59. doi: 10.1002/1099-0534(2000)12:5<321::AIDCMR4>3.0.CO;2-J.

6) de Vocht F, Muller F, Engels H, Kromhout H. Personal exposure to static and time - varying magnetic fields during MRI system test procedures. J Magn Reson Imag. 2009;30(5):1223-8. doi: 10.1002/jmri.21952. PMID: 19856458.

7) Schaap K, Christopher-De Vries Y, Crozier S, De Vocht F, Kromhout H. Exposure to static and timevarying magnetic fields from working in the static magnetic stray fields of MRI scanners: a comprehensive survey in the Netherlands. Ann Occup Hyg. 2014:meu057. doi: 10.1093/annhyg/meu057. PMID: 25139484.

8) Karpowicz J, Gryz K. Health risk assessment of occupational exposure to a magnetic field from magnetic resonance imaging devices. Int J Occup Saf Ergon.2006;12(2):155-67. doi: 10.1080/10803548.2006.11076679. PMID: 16790173.

9) Yamaguchi-Sekino S, Sekino M, Ueno S. Biological effects of electromagnetic fields and recently updated safety guidelines for strong static magnetic fields. Magn Resone Med Sci. 2011;10(1):1-10. doi: 10.2463/mrms.10.1. PMID: 21441722.

10) Foster KR, Glaser R. Thermal mechanisms of interaction of radiofrequency energy with biological systems with relevance to exposure guidelines. Health Phys. 2007;92(6):609-20. doi: 10.1097/01.HP.0000262572.64418.38. PMID: 17495663.

11) Wilén J, de Vocht F. Health complaints among nurses working near MRI scanners A descriptive pilot study. Eur J Radiol. 2011;80(2):510-3. doi: 10.1016/j.ejrad.2010.09.021. PMID: 20950976.

12) Kunt H, Dayığlu H. The Effects of Radiation on Bone Mineral Density of Radiology Workers Depending on The Device They Use. Eur J Gen Med. 2011;8(4):318-22.

13) Ali MA. Magnetic resonance imaging and associated alteration in some biophysical properties of blood. Rom J Biophys. 2007;17(4):277-86.

14) Bolton VN, Hawes SM, Taylor CT, Parsons JH. Development of spare human preimplantation embryos in vitro: an analysis of the correlations among gross morphology, cleavage rates, and development to the 
blastocyst. Journal of in vitro fertilization and embryo transfer. 1989;6(1):30-5. doi: 10.1007/BF01134578. PMID: 2708875.

15) Wdowiak A, Wdowiak L, Wiktor H. Evaluation of the effect of using mobile phones on male fertility. Ann Agr Environl Mede. 2007;14(1):169-72. PMID: 17655195.

16) Saadeldin IM, Fadel AM, Hamada MM, El-Badry AA. Effects of exposure to $50 \mathrm{~Hz}, 1$ Gauss magnetic field on reproductive traits in male albino rats. Acta Vet Brno. 2011;80(1):107-11. doi: 10.2754/avb201180010107.

17) Desai NR, Kesari KK, Agarwal A. Pathophysiology of cell phone radiation: oxidative stress and carcinogenesis with focus on male reproductive system. Reprod Biol Endocrinol. 2009;7(114):1-9. doi: 10.1186/1477-7827-7-114. PMID: 19849853, PMCID: PMC2776019.

18) Challis L. Mechanisms for interaction between RF fields and biological tissue. Bioelectromagnetics. 2005;26(S7):S98-S106. doi: 10.1002/bem.20119. PMID: 15931683.

19) Lantow M, Schuderer J, Hartwig C, Simko M. Free radical release and HSP70 expression in two human immune-relevant cell lines after exposure to $1800 \mathrm{MHz}$ radiofrequency radiation. Radiat Res. 2006;165(1):88-94. doi: 10.1667/RR3476.1. PMID: 16392966.

20) Kesari KK, Behari J. Evidence for mobile phone radiation exposure effects on reproductive pattern of male rats: role of ROS. Electromagn Biol Med. 2012;31(3):213-22. doi: 10.3109/15368378.2012.700292. PMID: 22897402.

21) Hamada AJ, Singh A, Agarwal A. Cell phones and their impact on male fertility: fact or fiction. Open Reprod Sci J. 2011;5(4):125-37. doi: 10.2174/1874255601103010125.

22) Agarwal A, Singh A, Hamada A, Kesari K. Cell phones and male infertility: a review of recent innovations in technology and consequences. International Braz J Urol. 2011;37(4):432-54. doi: 10.1590/S167755382011000400002. PMID: 21888695.

23) Zini A, Libman J. Oxidative stress and male infertility. Systems Biology of Free Radicals and Antioxidants: Springer; 2014. p. 2815-33. doi: 10.1007/978-3-642-30018-9_180.

24) Tremellen K. Oxidative stress and male infertility-a clinical perspective. Hum Reprod Update. 2008;14(3):243-58. doi: 10.1093/humupd/dmn004. PMID: 18281241.

25) De Iuliis GN, Newey RJ, King BV, Aitken RJ. Mobile phone radiation induces reactive oxygen species production and DNA damage in human spermatozoa in vitro.PLOS One. 2009; 4(7):e6446. doi: 10.1371/journal.pone.0006446. PMID: 19649291. PMCID: PMC2714176.

26) Ghanbari M, Mortazavi SB, Khavanin A, Khazaei M. Simultaneous effects of exposure to microwaves and noise on male rat's sperm parameters and total antioxidant capacity. Health Scope. 2013;1(4):180-6. doi: 10.17795/jhealthscope-8230.

27) Mollasadeghi A, Mehrparvar AH, Atighechi S, Davari MH, Shokouh P, Mostaghaci M, et al. Sensorineural hearing loss after magnetic resonance imaging. Case rep radiol. 2013. doi: 10.1155/2013/510258. PMID: 23844303.

28) Pramanik P, Biswas S. Traffic noise: a silent killer of male gamete of albino rats. Al Ameen J Med Sci. 2012;5(1):82-9.

29) El-Gebaly RH, El-Bialy NS, Rageh MM. A $50 \mathrm{~Hz} 0.5 \mathrm{mT}$ magnetic field induces cytogenetic effects and biological alterations in Wistar rat. Life Sci J. 2012;9(4).

30) Bernabò N, Tettamanti E, Russo V, Martelli A, Turriani M, Mattoli M, et al. Extremely low frequency electromagnetic field exposure affects fertilization outcome in swine animal model. Theriogenology. 2010;73(9):1293-305. doi: 10.1016/j.theriogenology.2009.12.010. PMID: 20176397. 